\title{
The research on edge detection algorithm of lane
}

\author{
Zhong-xun Wang ${ }^{*}$ and Wenqi Wang
}

\begin{abstract}
In developed countries, the automation and intelligent development of vehicles has reached a relatively high level and has gradually developed in China. In recent years, algorithms for lane detection have emerged in an endless stream, but the advantages and disadvantages of comprehensive comparison of various algorithms have resulted in the following problems: First, the robustness of lane line detection is poor, mainly due to the surrounding environment of the road. The impact is greater, in traffic-intensive city streets, affected by natural factors such as trees or building shadows around the driveway; second, the real-time nature of the lane line detection is poor, affected by other marking lines on the driveway, or damaged in the lane. When the pollution is serious, the image of the lane line collected by the system is incomplete and of poor quality, which increases the difficulty of analyzing and processing data of the detection system. This article aims at the problems existing in the current lane line detection and determines the research focus of the article: (1) Improve the accuracy and real-time performance of the detection algorithm. Most of the factors affecting the detection of the lane line are generated before the image is acquired. This requires the strict pre-processing of the collected lane line image to remove a large amount of interference. After the information, not only can the detection result be more accurate but also the complexity of the algorithm be simplified, making the detection result accurate and effective. (2) Use the FPGA for verification. This paper simulates the detection algorithm from two aspects of MATLAB and FPGA, learns the working mode of the related chip and different interface protocols, optimizes the logic design through the hardware design language, and facilitates the hardware implementation of the algorithm. This method can effectively improve the image processing speed, save the logic resources, and better realize the lane recognition function. (3) Improve the existing lane line detection algorithm. There are many algorithms for lane detection, but each algorithm has its own advantages and disadvantages. Part of this article summarizes the advantages and disadvantages of these detection algorithms, analyzes their feasibility in actual detection, and improves the algorithm based on this.
\end{abstract}

Keywords: Lane line detection and identification, Kirsch algorithm, MATLAB

\section{Introduction}

The automotive industry is one of the largest and most important industries in the world. According to statistics from China Association of Automobile Manufacturers in 2016, the total number of automobile production and sales in China was 28.119 million vehicles and 28.058 million vehicles, a record high [1].

With the constant popularity of automobiles and the continuous improvement of people's living standards, almost every family owns one or more cars, providing people with a comfortable and convenient travel life. Although entering the twenty-first century, the post-modern transportation

\footnotetext{
* Correspondence: ytuwwq@126.com

Institute of Science and Technology for Opto-Electronics Information, Yantai University, Yantai 264005, Shandong, China
}

system has been very developed, but it is still not in direct proportion to the increasing traffic environment demand of people. The rapid development of the automotive industry has also brought with it environmental pollution, energy shortages, traffic jams, and traffic safety issues, among which traffic safety issues are particularly acute and thorny and have developed into a major global problem [2].

In China, the number of casualties caused by road traffic safety exceeds 200,000 each year, and the total number of traffic accidents handled is approximately 4.7 million people [3]. Research shows that the number of casualties and property losses caused by traffic accidents will continue to increase substantially in the coming years, and the losses caused by road traffic will become one of the three major factors leading to global disease 
and injury in 2020 [4]. The causes of comprehensive traffic accidents mainly include four aspects: the car's own performance, drivers, roads, and environment. Among these four aspects, the driver's personality, fatigue driving, and drunk driving are the most common causes of accidents. When the car is in motion, the driver dominates, and the driver's driving operation directly determines the stability and safety of the vehicle during driving.

According to the statistics of traffic accidents, the human factor accounted for $95 \%$ of all the factors that caused the accident, and the accident rate caused by the driver's misoperation was as high as $73 \%$. This further indicates that the driver's misoperation is a traffic accident and the main factor that occurs [5].

Faced with such severe traffic safety problems, the development of vehicle safety technology has become an important research topic in order to reduce the number of people injured or killed in car accidents.

The current advanced car safety technology is mainly through computer, automatic control, and information fusion methods to improve the driver's driving safety factor, making the car more intelligent in the driving process. As a result, research on intelligent transportation systems began to develop. Based on the intelligent perception of the vehicle's driving environment, when the driver operates improperly, the system will automatically warn the driver through voice and other forms to correct the current erroneous driving operation. The system will also automatically replace the driver's control of the vehicle in special circumstances such as the driver's sleep or poor mental status, thereby avoiding traffic accidents.

Smart cars occupy the leading position in the intelligent transportation system. It not only includes the function of sensing environment and decision-making planning but also completes multi-level assisted driving functions. It is a brand new comprehensive high-tech system [6, 7]. Relying on its own sensors, smart cars can fully perceive the surrounding environmental information, which is a prerequisite for other functions.

However, there are no two identical roads in the world. This is because there are too many influencing factors on the road conditions. When natural and non-natural factors change, it is easy for drivers to make wrong driving judgments on specific road condition information which leads to traffic accident. If the vehicle can be judged incorrectly by the driver at this time, the correct judgment of the road conditions and timely warning to guide the driver's wrong operation will be able to effectively avoid the occurrence of traffic accidents. At this time, the smart car system is required to accurately judge the road conditions and feedback correct driving information to the driver [8]. Unmanned vehicles have already begun trial operation on a global scale. How to detect and identify road signs, obstacles, and pedestrians in an unknown environment in an accurate and real-time manner is the focus of the current research on unmanned vehicles [9].

To sum up, the in-depth study of lane detection and recognition technology has important application value and practical significance.

\section{Difficulty and insufficiency of lane detection}

Real-time and robustness are the purpose of lane detection. When the vehicle is driving at a certain speed, the road image collected must be processed in time to ensure the normal driving of the vehicle. The entire lane line detection system not only needs to adapt to the complicated and changeable road environment but also adapts to the influence of some force majeure factors. The specific conditions are summarized as follows:

1. Most existing lane line detection algorithms are mainly designed for structured roads and not structured roads, due to the influence of the surrounding environment of the road, such as the influence of shadows of surrounding trees or buildings and the pair of vehicles ahead of the road. The influence of the lane line shielding, etc. makes the detection of the lane line very poor.

2. When affected by other markers on the driveway, or when the lane is damaged or the pollution is serious, the image of the lane line collected by the system is incomplete and of poor quality, making it difficult for the system to accurately judge and analyze the data, and it is impossible to guarantee the correctness of the data and real-time detection of lane lines.

Unclear detection and multi-peak detection problems are the biggest problems faced by traditional Hough transforms and are severely affected by noise. After the Hough transform is improved, the robustness and adaptability of the detection result are enhanced. In order to overcome the weather changes and the camera lens is not clean, this paper uses the two-dimensional median filtering method to process the original image.

In the edge detection stage, the Kirsch algorithm has obvious superiority, and this paper upgrades on the basis of the original classical algorithm, which makes the image processing speed more than twice the original, expands the scope of use, and improves the detection and identification of lane lines. Speed, and while speeding up, does not affect the detection of lane lines.

\section{Demand analysis of lane detection and identification}

The detection and identification of the lane line is to realize the intelligence and automation of the modern 
transportation system. It has a good application in the fields of advanced parking assistance, lane keeping assistance, off-road warning, and lane change assistance.

Demand analysis is a necessary step in system design. It can not only understand the operating mechanism of the system before design but also adjust the design structure in time for the demand. For the lane line identification, the ultimate goal is to accurately and efficiently identify the lane line. For this purpose, do the following needs analysis:

\section{(1) Accuracy}

The purpose of intelligent transportation system design is to reduce the rate of urban traffic accidents, so accuracy is the primary requirement of system design. If there is an error or deviation in the detection of the lane line, it may cause the vehicle to head in the wrong direction, which is not conducive to its own driving safety and brings greater safety risks to road safety.

(2) High efficiency

When the vehicle is driving on the road, it must not only be able to accurately follow the direction of the lane line but also be able to maintain a certain speed. This requires both the detection accuracy and the detection in the lane line identification process efficiently and in real-time.

\section{(3) Memory}

In the intelligent public transport system, in addition to the vehicle that can detect the lane line image in real time, it also has a good memory function, which can effectively store and manage the recorded data information and facilitate investigation and evidence collection after a traffic accident.

\section{(4) Simple design structure}

The system design is mainly used in vehicles, and the occupied space should be as small as possible. You can make full use of $5 \mathrm{G}$ communication real-time connection without affecting other functions of the vehicle.

(5) Fully automated
The system is designed to allow the driver to alert and correct the driver's driving errors in an unconscious situation, so it is necessary to be able to fully automate the system during operation.

\section{The proposed method-lane line detection and identification}

The lane detection and recognition process consists of the following components: image acquisition, image preprocessing, image segmentation, edge detection, feature extraction, feature point identification, and lane line recognition, as shown in Fig. 1.

\subsection{Road image acquisition and pretreatment}

In the lane line detection process, the image processed by the system is mainly captured by the car's camera. The pictures used in this article are mainly shot on a Canon EOS 100D DSLR camera. In the smart car identification and navigation system based on image processing, due to the interference of external factors in the actual road conditions, such as the influence of optical fiber, weather, roads, and the surrounding environment, the images collected by the smart car camera include spots, pits, etc. The interference factors and the decline of image quality will directly affect the detection and recognition of the target information lane line [10]. Therefore, it is necessary to preprocess the collected images, reduce the useless interference information and enhance the target information, simplify the image processing algorithm, and improve the detection and recognition accuracy of lane lines.

The image of the lane line is taken from the real scene shooting. The speed of the vehicle traveling on the road is relatively fast. When shooting the lane line image, it can only be shot on the zebra crossing when the red light is on. For safety reasons, consider turning left and right. The vehicle needs to be fast and accurate when shooting. This camera is also chosen to achieve better focus in a short time, improve focus speed, and ensure image quality.

Due to the influence of actual road conditions and other external disturbance factors, the images collected by the smart car camera contain various interference factors such as spots and pits, and the degradation of image quality will directly affect the detection and

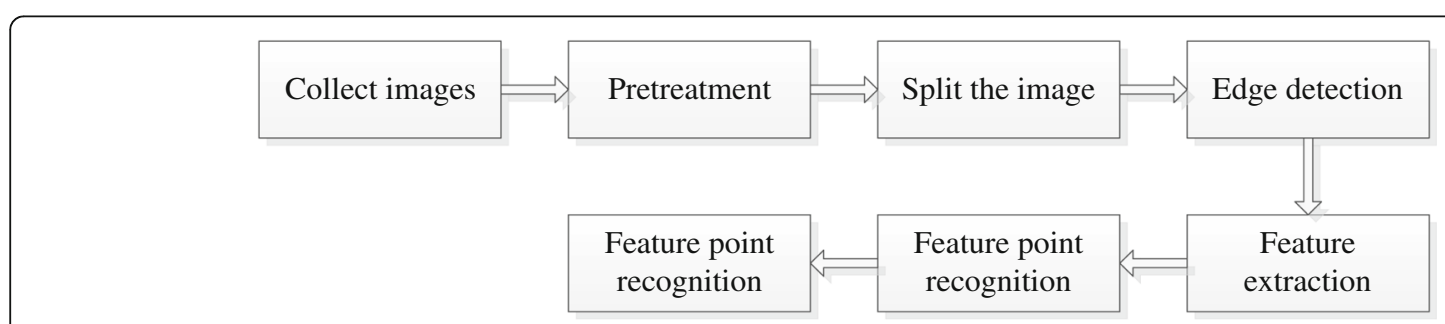

Fig. 1 Lane detection and recognition process 
recognition of the target information lane line. Therefore, image preprocessing is a necessary step before analyzing and judging image information.

The purpose of image preprocessing is to remove the noise in the image, save the useful road surface information reasonably, minimize the useless information in the road surface information, and strengthen the target information, thereby simplifying the algorithm complexity of image processing.

The image preprocessing part of this paper mainly includes road image graying, median filter method noise reduction, and image edge enhancement. Image graying reduces the amount of color information in the image and simplifies the complexity of the algorithm; two-dimensional wavelet packet decomposition technology can adaptively select the frequency band and improve the resolution of the frequency band; and image enhancement highlights the characteristics of the region of interest and can enhance the grayscale of the image. In contrast, lane line information strengthens.

The image captured by the smart car camera is a color image containing a lot of extraneous information. Because the grayscale image contains less information, the lane line detection algorithm usually directly processes the grayscale image to speed up the processing speed of the later detection algorithm. In the RGB color space, the color of the image is different according to the three primary colors and proportional mixing results [11]. To convert an RGB color image to a grayscale image, the luminance value is quantized. There are generally four methods for graying a color image: the average method, the component method, the maximum method, and the weighted average method. The weighted average method is selected here for graying. Equation (1) shows the transformation relationship, and the processed lane line effect diagram is shown in Fig. 2.

$$
\left\{\begin{array}{c}
V_{\text {gray }}=0.290 R+0.587 G+0.116 B \\
V_{\text {gray }}=R=G=B
\end{array}\right.
$$

Salt and pepper noise is the most common and main source of noise in lane line images. It is generally characterized by black-white impulse noise points, which tends to blur the road image and flood the feature information, which brings great difficulties to subsequent processing. Therefore, it is necessary to filter out noise to improve image quality. The principle of image filtering processing is to preserve the original useful information in the image while removing the noise. After comparing various filtering methods, it is found that the median filtering has the best filtering effect on the salt and pepper noise, which can overcome the edge blurring phenomenon and can be better, preserve original image contour information, and has good real-time performance.

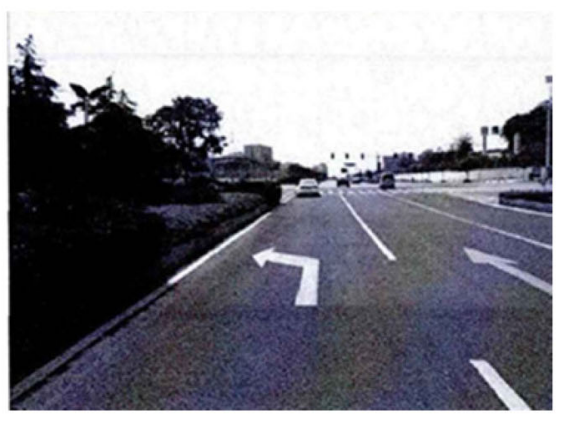

(a)

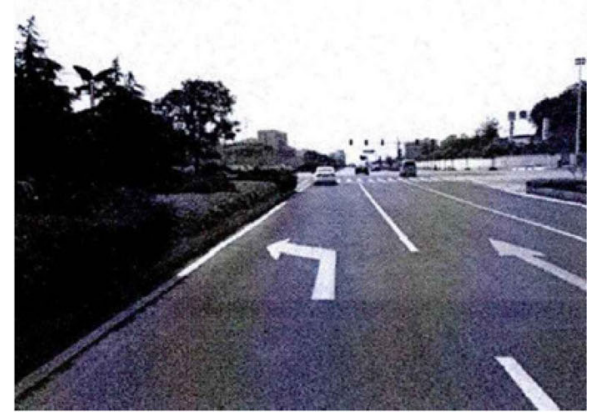

(c)

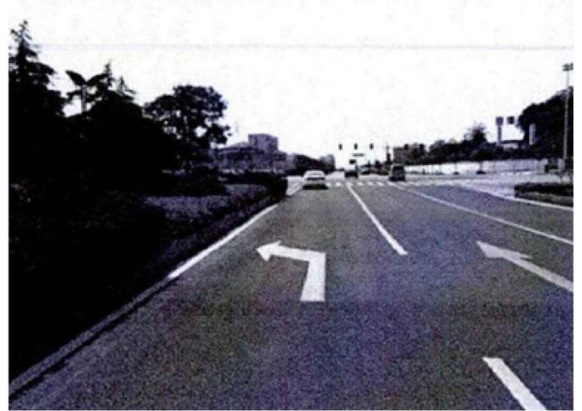

(b)

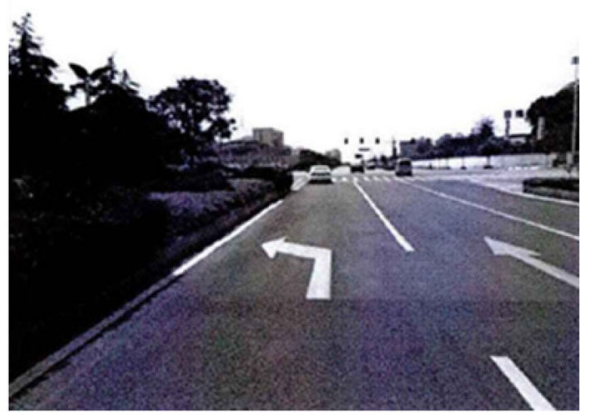

(d)

Fig. 2 After the processing of the lane line effect map. a Salt and pepper noise. b Mean filter image. c Gaussian filter image. d Median filter image 
After filtering, there is still a lot of interference information for lane lines to be identified. The main purpose of the image edge enhancement is to emphasize the contour information of the lane line as much as possible based on the grayscale features of the lane line edge in the grayscale image and to reduce the interference caused by other non-lane line object contours $[12,13]$. After image edge enhancement, detection and recognition of subsequent lane lines are made more accurate. The comparison of different enhancement methods shows that the Laplacian-based image enhancement method can not only enhance the high-frequency components of the image but also preserve the low-frequency components of the image, which is an effective method for image edge enhancement [14].

\subsection{Lane line edge detection}

There are many types of road traffic signs in China. The regulations of the People's Republic of China Traffic Road Marking Line clearly define the color and shape characteristics of China's traffic signs. In terms of color, there are two kinds of pure white and pure yellow. The pure white marking line is divided into white solid line and white dashed line to distinguish different lanes in the same direction. The pure yellow marking line is further divided into double yellow line, single yellow line, yellow solid line, yellow dotted line, etc., which are used to distinguish different directions of the lane; in terms of shape, for the lane line, it is mainly divided into solid line, dotted line, and straight line and so as for the curve two.
In the process of actually detecting the lane line, it will be affected by other traffic signs, light, and color fading of the road. However, according to the actual image collected, the color difference between the lane line and the road surface is still obvious, as shown in Fig. 3. Therefore, the color can be used as one of the feature information for detecting the lane line.

The edge of the image is the singularity and mutation point of the grayscale function of the image, that is, the area of the image where the grayscale information changes drastically. Along the pixels at the edges of the image, the gray value changes slowly, and the pixels that are perpendicular to the edge direction have drastically changed gray values. The nighttime road surface image is affected by uneven illumination, so it is not suitable to use a special threshold segmentation algorithm to obtain binary images. The edge detection algorithm can obtain binary images without using a special threshold segmentation algorithm. The edge of the object is the region where the local brightness of the image changes significantly. The identification and extraction of the image edge is very important for the recognition and understanding of the entire image scene. It is also an important feature that the image segmentation depends on. The commonly used edge detection operator is the Sobel operator, LOG operator, Kirsch operator, Canny operator, etc. [14]. Various algorithms have advantages and disadvantages for different occasions. The purpose of this paper is to explore an algorithm that can detect

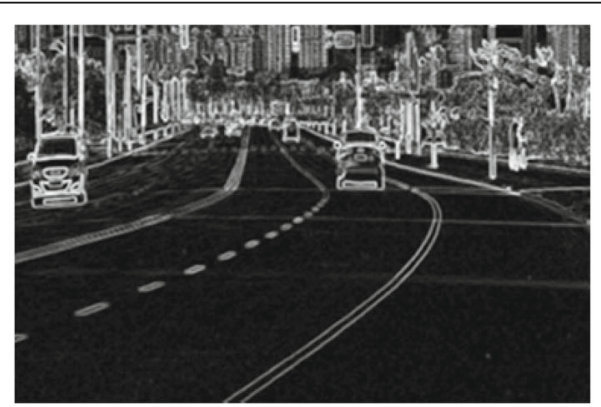

(a)

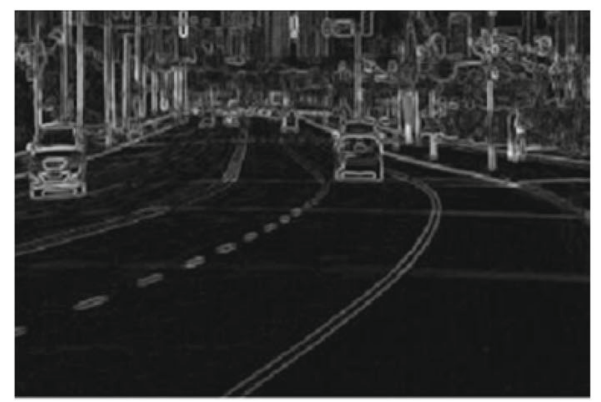

(c)

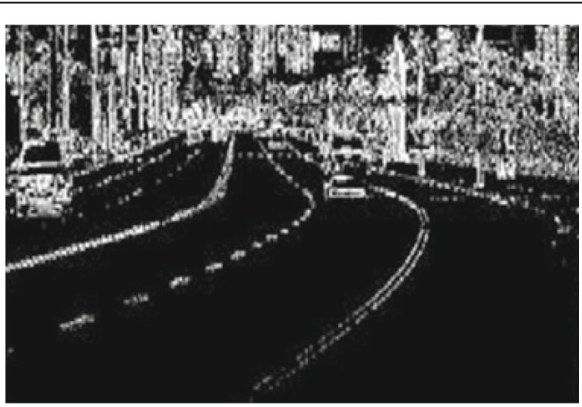

(b)

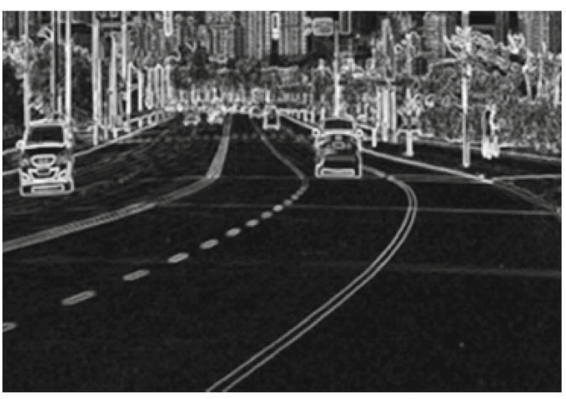

(d)

Fig. 3 Comparison of test operator results. a Sobel operator. b LoG operator. c Kirsch operator. $\mathbf{d}$ Canny operator 
clear lane line edge images and quickly and efficiently identify images.

Combined with the principle of each detection algorithm, a comparative analysis is performed using the curve image as an example and Matlab simulation is performed. The result chart is shown in Fig. 3.

Through the comparison of the above groups of inspection charts, it is not difficult to find that the Kirsch detection operator has obvious advantages, the most abundant information extraction, and the best detection results. However, the Kirsch algorithm itself also has its own deficiencies, such as large amount of computation, real-time, and poor validity. For these problems, this paper will improve and upgrade the algorithm in the next section and use this algorithm to detect edge information in the entire system.

\subsection{Lane line feature point recognition}

Based on the road model recognition method, the straight line model is the simplest one. This paper mainly introduces the Hough transform to detect straight lines.

The Hough transform [15] was proposed by Paul Hough in 1962 and published as a patent in the USA. Its principle is to convert the detection problem from the image space to the parameter space and find the peak of the accumulator in the parameter space so as to achieve the target detection.

The specific detection algorithm is as follows:

In the image $x-y$ coordinate space, the line passing through point $\left(x_{j}, y_{j}\right)$ is represented by:

$$
y_{j}=u x_{j}+v
$$

Among them, parameter $u$ is the slope, and $v$ is the intercept.

If $\mathrm{x}_{j}$ and $y_{j}$ are treated as constants and parameters, $u$ and $v$ are treated as variables, then Eq. (2) can be expressed as:

$$
v=-x_{j} u+y_{j}
$$

The other point of $\left(x_{k}, y_{k}\right)$ in the coordinate space also has a corresponding line in the parameter space:

$$
v=-x_{k} u+y_{k}
$$

This line intersects the line of point $\left(x_{j}, y_{j}\right)$ in the parameter space at point $\left(u_{0}, v_{0}\right)$ as shown in Fig. 4:

It is known that when the slope of a straight line is infinite or nearly infinite, it cannot be expressed in the parameter space, so the polar coordinate system is used next, and the polar coordinate expression is as follows:

$$
\rho=x * \cos \theta+y * \sin \theta
$$

Where $\rho$ is the vertical distance from the straight line to the origin and $\theta$ is the angle from the $x$ axis to the vertical line. The value range is $\pm \pi / 2$. When $\theta=0^{\circ}, \rho$ is equal to the intercept of the $x$ axis. When $\pm \pi / 2, \rho$ is the cutoff of the $y$ axis distance.

The point on the line in the $x-y$ plane is mapped to the parameter space as a sinusoid. As shown in Fig. 5c, the line equation can be determined by the intersection of the two sinusoids.

In the actual calculation, the Hough transform builds a two-dimensional accumulative array based on the parameter space by accumulating the curve values in the $\rho-\theta$ parameter space [16]. Here, the quantization accuracy of 1 or 0.5 is selected to quantize the variables $\rho$ and $\theta$ as the two-dimensional array. The value of each cell represents the number of collinear points in the image. Therefore, the conversion of the straight line parameter is converted to the peak point in the search.

The Hough transform detects lane lines with good self-adaptability. When the lane lines are not continuous, it can still detect the effect very well and has good robustness.

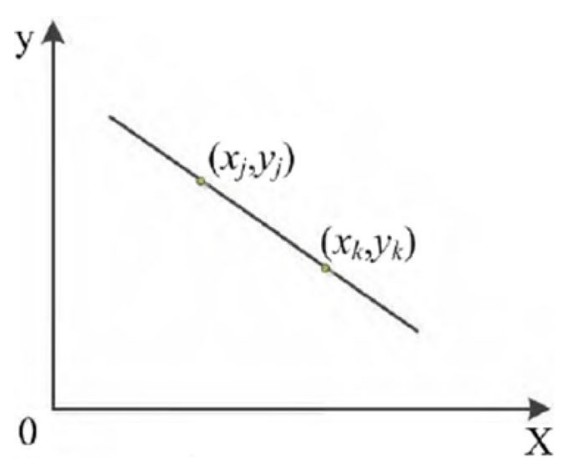

(a)

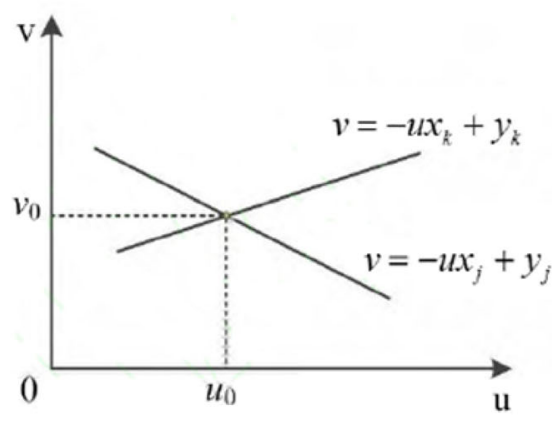

(b)

Fig. 4 Hough transform in Cartesian coordinates. a Image coordinate space. b Parameter space 


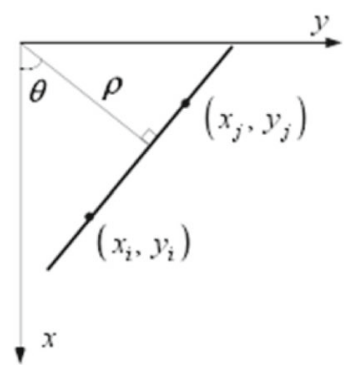

(a)

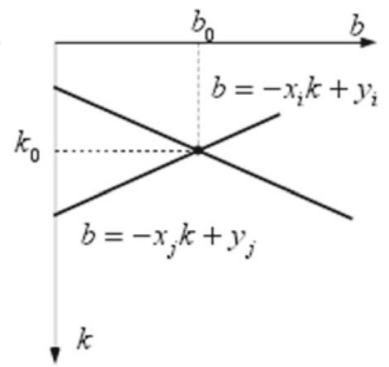

(b)

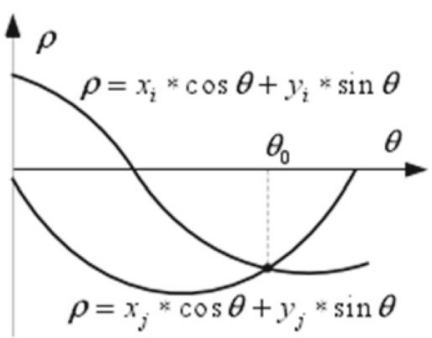

(c)

Fig. $5 x-y$ space and corresponding parameter space in polar coordinate system. $\mathbf{a} x-y$ space. $\mathbf{b} k-b$ space. $\mathbf{c} \quad-\theta$ space

\section{Algorithm improvement and upgrading-discussion and results}

Edge is the most basic feature of the image. Due to grayscale discontinuities and other reasons, differential operators in these places are often relatively large. Therefore, when using such operators for detection, multi-directional differential operators are generally used for processing. The classical Kirsch operator edge detection is to use eight direction templates to detect the image and then select the maximum value as the image edge. However, the computational complexity of processing images through eight detection templates is rather large, and it cannot meet the real-time and effectiveness of detection to some extent. For computational problems, this paper improves and upgrades the classical Kirsch operator, which effectively reduces the amount of computation [17].

The Kirsch operator is a new edge detection algorithm proposed by R. Kirsch. It uses a template in eight different directions to perform convolutional derivation of each pixel in the target image, so as to obtain a complete image edge information [14]. The specific principle is as follows:

Assume that $M_{n}(i, j)$ is the value at $(i, j)$ in the $n$th filter template, $I$ represents the input image, and $I(\mathrm{x}, \mathrm{y})$ represents the pixel value at the point $(x, y)$ in the image. First, design the eight-direction filter template and use these eight templates to filter the target image $[18,19]$. Set the filtering value at the point $(x, y)$ obtained by $n$ template filters to be $f_{n}(x, y)$. The value definition is as follows:

$$
f_{n}(x, y)=\sum_{(i, j) \in \sigma} I(x+i, y+j) M_{n}(i . j)
$$

where $\sigma$ represents the filter template $3 \times 3$ region, $i=-1,0,1 ; j=-1,0,1 ; x=1,2, \ldots, H ; y=1,2, \ldots W, H$, and $W$ respectively represents the height and width of the image I.

The largest filter value is:

$$
f_{\max }(x, y)=\max \left\{f_{1}(x, y), f_{2}(x, y), \cdots, f_{8}(x, y)\right\}
$$

Then the extreme point image $F_{\max }$ can be expressed as follows:

$$
F_{\max }=\left(\begin{array}{ccc}
F(1,1) & \cdots & F(1, \mathrm{~W}) \\
\vdots & \ddots & \vdots \\
F(H, 1) & \cdots & F(H, W)
\end{array}\right)
$$

Among them, $F(x, y)$ represents the gray value at the filtered point $(x, y)$. The edge image $E$ is obtained from the extreme point image $F_{\max }$ and the preset threshold $T$, defined as follows:

$$
\mathrm{E}=\left(\begin{array}{ccc}
E(1,1) & \cdots & E(1, \mathrm{~W}) \\
\vdots & \ddots & \vdots \\
E(H, 1) & \cdots & E(H, W)
\end{array}\right)
$$

where $E(x, y)$ denotes the pixel value at $(x, y)$ in image $E$, when $F(x, y)>T, E(x, y)=1$, otherwise $E(x, y)=0$.

In the actual road image detection, because the lane lines are only in four directions, the four templates are used to filter the target image. The specific template selection is as follows:

$$
\begin{array}{rlrl}
M_{1} & =\frac{1}{8}\left(\begin{array}{ccc}
-2 & -1 & 0 \\
-1 & 0 & 1 \\
0 & 1 & 2
\end{array}\right) & M_{2}=\frac{1}{8}\left(\begin{array}{ccc}
2 & 1 & 0 \\
1 & 0 & -1 \\
2 & 1 & 0
\end{array}\right) \\
M_{3}=\frac{1}{8}\left(\begin{array}{ccc}
1 & 1 & 2 \\
-1 & 0 & 1 \\
-2 & -1 & 0
\end{array}\right) & M_{4}=\frac{1}{8}\left(\begin{array}{ccc}
0 & -1 & -2 \\
1 & 0 & -1 \\
2 & 1 & 0
\end{array}\right)
\end{array}
$$

The Kirsch operator is composed of eight $3 \times 3$ window templates, each representing a specific detection [6]. As follows:

$$
\begin{aligned}
& \left(\begin{array}{ccc}
5 & 5 & 5 \\
-3 & 0 & -3 \\
3 & -3 & -3
\end{array}\right)\left(\begin{array}{ccc}
5 & 5 & -3 \\
5 & 0 & -3 \\
-3 & -3 & -3
\end{array}\right)\left(\begin{array}{ccc}
5 & -3 & -3 \\
5 & 0 & -3 \\
5 & -3 & -3
\end{array}\right)\left(\begin{array}{ccc}
-3 & -3 & -3 \\
5 & 0 & -3 \\
5 & 5 & -3
\end{array}\right) \\
& \left(\begin{array}{ccc}
-3 & -3 & -3 \\
-3 & 0 & -3 \\
5 & 5 & 5
\end{array}\right)\left(\begin{array}{ccc}
-3 & -3 & -3 \\
-3 & 0 & 5 \\
-3 & 5 & 5
\end{array}\right)\left(\begin{array}{ccc}
-3 & -3 & 5 \\
-3 & 0 & 5 \\
-3 & -3 & 5
\end{array}\right)\left(\begin{array}{ccc}
-3 & 5 & 5 \\
-3 & 0 & 5 \\
-3 & -3 & -3
\end{array}\right)
\end{aligned}
$$

At this point, according to the classical algorithm, the number of addition operations is $P=7$ times, the number 
of multiplication is $M=2$ times, the number of addition in each direction is $7 \times 8=56$ times, and the number of multiplication is $2 \times 8=16$ times. Therefore, for an $N \times M$ image, the number of addition operations needed to improve the algorithm is $P=56 N^{2}$ times, and the number of multiplication operations is $M=16 N^{2}$ times.

Suppose that the eight template factors are arranged in a clockwise direction into matrix $M$, then $M$ is a cyclic matrix, and the value of each row element is shifted to get the value of the next line element [19-22]. The transformation of $M$ is carried out that:

$$
\begin{gathered}
B M=C \\
\text { 令 } P=\left(\mathrm{p}_{0}, \mathrm{p}_{1}, \mathrm{p}_{2}, \cdots, \mathrm{p}_{T}\right)^{T} \\
A=\frac{1}{8}\left(\mathrm{q}_{0}, \mathrm{q}_{1}, \mathrm{q}_{2}, \cdots, \mathrm{q}_{T}\right)^{T}=\left(\mathrm{r}_{0}, \mathrm{r}_{1}, \mathrm{r}_{2}, \cdots, \mathrm{r}_{T}\right)^{T}
\end{gathered}
$$

Then

$$
\mathrm{MP}=8 \mathrm{~A}
$$

Through (11) and (12) can get

$$
\mathrm{B}^{-1} C P=8 A, C P=8 A B
$$

Expand (13) and move by:

$$
\begin{aligned}
& r_{0}=0.625\left(p_{0}+p_{1}+p_{2}\right)-0.375\left(p_{3}+p_{4}+p_{5}+p_{6}+p_{7}\right) \\
& r_{1}=r_{0}+p_{7}-p_{2} \\
& r_{2}=r_{1}+p_{6}-p_{1} \\
& r_{3}=r_{2}+p_{5}-p_{0} \\
& r_{4}=r_{3}+p_{4}-p_{7} \\
& r_{5}=r_{4}+p_{3}-p_{6} \\
& r_{6}=r_{3}+p_{2}-p_{5} \\
& r_{7}=r_{6}+p_{1}-p_{4}
\end{aligned}
$$

Calculate the number of operations required for (4) $P=7+2 \times 7=21$ times, the number of multiplication operations $M=2$ times; so for an $N \times M$ image, the improved algorithm required to add the number of operations is $P=21 N^{2}$ times, multiplication times for the $M=3 N^{2}$ times.

It can be concluded that the algorithm has reduced the amount of addition and multiplication operations, and it reduces the amount of calculation while improving the effectiveness and real-time performance of the entire system. The improved effect of the algorithm is shown in Fig. 6:

From the comparison chart, we can see that when the computational complexity of the operator is greatly reduced, it does not affect the detection effect and the edge detection is still good.

\section{Conclusions}

The development of intelligent transportation has promoted the research and development of lane line detection. The intelligent development process is also faced with many difficulties and deficiencies. This article is based on these difficulties and deficiencies, analyzes the causes of the line, and detects the edge of the lane line. The basic questions were systematically studied. Through the improved Hough transform and Kirsch operator, the robustness and adaptability of the detection results are enhanced, the redundant information of the operator is reduced, the computational complexity of the algorithm is reduced by the matrix operation, and the detection of lane lines is improved. Speed enhanced real-time. The development of the Internet of Things era will surely promote the development of smart vehicles to achieve new breakthroughs and innovations. We expect smart cars to approach the homes of ordinary people as soon as possible, bringing convenience, safety, and comfort to our lives.

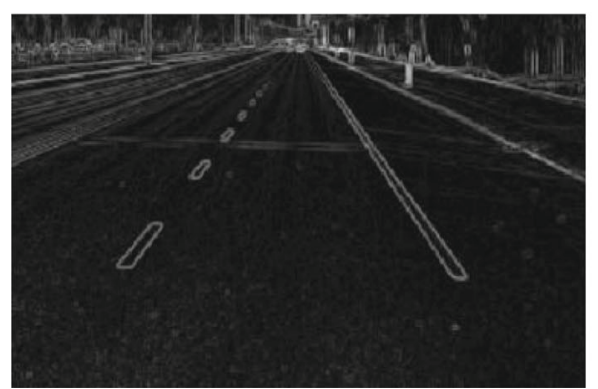

(a)

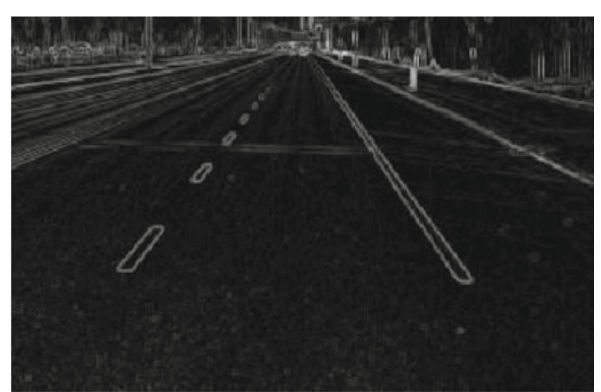

(b)

Fig. 6 Optimized Kirsch detection operator. a Before optimization. b Optimized 


\section{Abbreviations}

FPGA: Field Programmable Gate Array; MATLAB: Matrix laboratory; RGB: Red, green, and blue mode

\section{Authors' contributions}

ZW is the first author. He designed the study, performed the research. WW is the communication author. She helped the first author and collected the data. Both authors read and approved the final manuscript.

\section{Authors' information}

Zhongxun Wang, male, born in 1965, Ph.D, is a professor. His research direction is source channel coding in wireless communications. Wenqi Wang, born in 1992, is a master degree candidate of Yantai University and her research direction is mobile image processing and lane detection.

\section{Ethics approval and consent to participate}

Not applicable.

\section{Consent for publication}

Approved.

\section{Competing interests}

The authors declare that they have no competing interests.

\section{Publisher's Note}

Springer Nature remains neutral with regard to jurisdictional claims in published maps and institutional affiliations.

Received: 30 May 2018 Accepted: 28 August 2018

Published online: 01 October 2018

\section{References}

1. F. Yuwu, The year of 2016 is a year of transition. The future of China's auto industry is full of vitality. Metal Processing(Cold Work) 2017(z1), 2-3

2. K. Wang, Research on the detection method of obstacles in front of unmanned vehicles (Anhui University, 2012)

3. Anonymous, China has over 200,000 casualties per year due to traffic safety accidents. Practical Automotive Technology 8, 10 (2013)

4. M. Peden, R. Scurfield, D. Sleet, et al., World report on road traffic injury prevention. Medical Journal Armed Forces India 10(4), 255 (2004)

5. P. Liu, K. Jia, Research on Fast Algorithm for Video ROI Detection[J]. Journal of Software, 8(11), (2013)

6. X. Youchun, W. Rongben, L. Bing, et al., A review of the status of intelligent vehicles in the world. Automotive Engineering 23(5), 289-295 (2001)

7. R. Bishop. Intelligent vehicle applications worldwide. Intelligent systems \& their applications IEEE, 2000, 15(1):78-81

8. L. Tao, F. Yangyu, W. Xiaopeng, et al., Lane line detection algorithm based on morphological structural element modeling. Journal of Computer Applications 29(2), 440-443 (2009)

9. R.D. Bretherton, G.T. Bowen, INCIDENT DETECTION AND TRAFFIC MONITORING IN URBAN AREAS[J]. Annals of Surgical Oncology, 1991, 4(4):349-354

10. M.S. Shirazi, B. Morris, Observing behaviors at intersections: A review of recent studies \& developments[C]// Intelligent Vehicles Symposium. IEEE, 2015:1258-1263

11. W.U. Zhi-Jun, S.L. Hong, B.J. Wang, Research on Lane Line Identification Algorithm Based on Sobel and hough Transform[J]. Computer Knowledge \& Technology, 2017.

12. F. Liming, L. Bijun, Y. Yutong, Lane detection based on multiscale resampling. Electronic Technology and Applications 43(4), 7-12 (2017)

13. C. Wuzhi, J. Yuting, T. Dongkui, et al., A fast lane recognition algorithm based on edge point projection. Automot. Eng. V39(3), 357-363 (2017)

14. M. Aly, Real time Detection of Lane Markers in Urban Streets[J]. Computer Science, 2014:7-12

15. A. Broggi, E.D. Dickmanns, IV urapidil is comparable to oral captopril for the treatment of patients with hypertensive urgency. Image \& vision Computing 18(5), 365-366 (2000)

16. K. Kluge, Extracting road curvature and orientation from image edge points without perceptual grouping into features[C]// Intelligent Vehicles '94 Symposium, Proceedings of the. IEEE, 2002:109-114
17. M. Bertozzi, A. Broggi, GOLD: a parallel real-time stereo vision system for generic obstacle and lane detection. IEEE Transactions on Image Processing A Publication of the IEEE Signal Processing Society 7(1), 62 (1998)

18. R. Tapia-Espinoza, M. Torres-Torriti, A comparison of gradient versus color and texture analysis for lane detection and tracking[C]// Robotics Symposium. IEEE, 2009:1-6

19. J.D. Crisman, C.E. Thorpe, SCARF: A color vision system that tracks roads and intersections. IEEE Trans Rob Autom 9(1), 49-58 (1993)

20. Y. Maeda, S. Suzuki, N. Kawasaki, IMAGE PROCESSING APPARATUS AND LANE PARTITION LINE RECOGNITION SYSTEM INCLUDING THE SAME:; US20160196474[P]. 2016

21. Q. Wang, J. Gao, H. Li, Grassmannian Manifold Optimization Assisted Sparse Spectral Clustering[C]// IEEE Conference on Computer Vision and Pattern Recognition. IEEE, 2017:3145-3153

22. B. Lu, G. Yue, X. Wang, et al., Factor-graph-based soft self-iterative equalizer for multipath channels. Eurasip Journal on Wireless Communications \& Networking 2005(2), 1-10 (2005)

\section{Submit your manuscript to a SpringerOpen ${ }^{\circ}$ journal and benefit from:}

- Convenient online submission

- Rigorous peer review

- Open access: articles freely available online

- High visibility within the field

- Retaining the copyright to your article

Submit your next manuscript at $\boldsymbol{\nabla}$ springeropen.com 